\title{
Intracellular Vacuoles in Experimental Acute Pancreatitis in Rats and Mice Are an Acidified Compartment
}

\author{
Claus Niederau and James H. Grendell \\ Department of Medicine, University of Düsseldorf, Federal Republic of Germany; Departments of Medicine and Physiology, University \\ of California, San Francisco, California 94143; and Medical Service, San Francisco General Hospital, San Francisco, California 94110
}

\begin{abstract}
The appearance of vacuoles inside acinar cells characterizes an early stage of development in different models of acute pancreatitis and, possibly, also in human disease. The vacuoles have been shown to contain both digestive and lysosomal enzymes. This abnormal admixture may have important implications for the pathogenesis of pancreatitis because the lysosomal enzyme cathepsin B can activate trypsinogen and may, by this way, trigger pancreatic autodigestion. For the activation process of trypsinogen by cathepsin B, however, an acidic pH is required. This study, therefore, looked for evidence of vacuole acidification in two different models of acute pancreatitis. Edematous pancreatitis was induced in rats by hyperstimulation with cerulein and hemorrhagic pancreatitis was induced in mice by feeding a choline-deficient, ethionine-supplemented diet. Pancreatic acinar cells were isolated at different times after induction of pancreatitis and incubated with $\mathbf{5 0}$ $\mu \mathrm{M}$ of acridine orange to identify acidic intracellular compartments. As shown in previous work, zymogen granules are the main acidic compartment of normal acinar cells; they remained acidic throughout the course of pancreatitis in both models. Vacuoles became increasingly more frequent in both models as pancreatitis progressed. Throughout development of pancreatitis, vacuoles accumulated acridine orange indicating an acidic interior. Addition of a protonophore (10 $\mu \mathrm{M}$ monensin or $5 \mu \mathrm{M}$ carbonyl cyanide m-chlorophenylhydrazone [CCCP] or a weak base ( $5 \mathrm{mM} \mathrm{NH} 4 \mathrm{Cl}$ ) completely and rapidly abolished acridine orange fluorescence inside both zymogen granules and vacuoles providing further evidence for an acidic interior. The acidification of vacuoles seen in two different models of pancreatitis may be an important requirement for activation of trypsinogen by cathepsin $B$ and thus for the development of acute pancreatitis.
\end{abstract}

\section{Introduction}

The appearance of vacuoles inside acinar cells characterizes an early stage of development in different models of acute pancreatitis (1-8) and possibly also in human disease (9). These

Address reprint requests to Dr. Niederau, Medizinische Klinik und Poliklinik, Abteilung für Gastroenterologie, Universität Düsseldorf, Moorenstrasse 5, 4000 Düsseldorf, FRG.

Presented in part at the Annual Meeting of the American Gastroenterological Association, San Francisco, CA, 18-21 May 1986, and has appeared in abstract form in 1986. (Gastroenterology. 90:1565.) 1987

Received for publication 8 January 1987 and in revised form 8 June

J. Clin. Invest.

(c) The American Society for Clinical Investigation, Inc.

0021-9738/88/01/0229/08 \$2.00

Volume 81, January 1988, 229-236 vacuoles have been shown to contain at the same time digestive and lysosomal enzymes suggesting abnormal intracellular processing of newly synthesized digestive and lysosomal enzymes in acute pancreatitis (5, 10-12). Physiologically, digestive and lysosomal enzymes are synthesized in ribosomes attached to the rough endoplasmic reticulum. They migrate through this compartment and are transported to the Golgi complex. During maturation of condensing vacuoles, the digestive and lysosomal enzymes diverge into zymogen granules and lysosomes. In the two models of pancreatitis used for the present experiments this normal routing of enzymes is altered. Both in hemorrhagic pancreatitis induced by feeding a choline-deficient, ethionine-supplemented (CDE) ${ }^{1}$ diet to mice, and in edematous pancreatitis induced by cerulein hyperstimulation in rats, normal secretion of digestive enzymes via exocytosis of zymogen granules is markedly decreased and intracellular vacuoles appear early in the course of pancreatitis (1-8, 10-12). The abnormal admixture of digestive and lysosomal enzymes inside the vacuoles may have important implications for the initiation of pancreatitis because is is known that the lysosomal hydrolase cathepsin B can activate trypsinogen in vitro (13). Activation of trypsinogen in vivo may trigger the autodigestive process in acute pancreatitis. Cathepsin B can activate trypsinogen, however, only at an acidic pH (13). Thus, the $\mathrm{pH}$ inside the vacuoles needs to be acidic for this activation process to occur. Therefore, the aim of this study was to look for evidence of vacuole acidification in the two models of acute pancreatitis. We also examined the acidification of zymogen granules under the pathological conditions of acute pancreatitis.

\section{Methods}

\section{Materials}

Hyaluronidase (type 1-S), alpha-chymotrypsin, collagenase (type 2139), soybean trypsin inhibitor (type 1-S), DNAse (type 1), N-2-hydroxyethylpiperazine- $N$-2-ethanesulfonic acid (Hepes), carbonyl cyanide $\mathrm{m}$-chlorophenylhydrazone (CCCP), monensin, bovine serum albumin were purchased from Sigma Chemical Co. (St. Louis, MO). Acridine orange (AO) was purchased from Kodak (Rochester, NY). CCCP and monensin were prepared in absolute ethanol stock solutions. Cerulein was purchased from Adria Laboratories (Columbus, $\mathrm{OH}$ ). The choline-deficient diet was purchased from Teklad (Madison, WI). DL-Ethionine (Sigma Chemical Co.) was mixed with the cholinedeficient diet to yield a final concentration of $0.5 \%$ ethionine (wt/wt).

Induction of experimental acute pancreatitis

Cerulein-induced pancreatitis. Male Sprague-Dawley rats weighing 200-300 g (Simonsen Laboratories, Gilroy, CA) were used in these

1. Abbreviations used in this paper: $\mathrm{AO}$, acridine orange; $\mathrm{CCCP}$, carbonyl dycyanide m-chlorophenylhydrazone; CDE, choline-deficient, ethionine-supplemented diet; HR, Hepes-Ringer buffer. 
studies. Rats were fed regular chow ad lib. throughout the experiments. All animals received seven intraperitoneal injections of $50 \mu \mathrm{g} / \mathrm{kg}$ body wt cerulein in hourly intervals over a 6-h period. This treatment with a supramaximal dose of the cholecystokinin (CCK)-analogue cerulein results in a rapid decrease of normal ductal pancreatic secretion, in an increase in pancreatic enzymes in serum, and the morphological signs of an acute edematous pancreatitis (2-5). Intracellular vacuoles are one of the earliest morphological alterations and start to appear during the first $3 \mathrm{~h}$, after the beginning of the cerulein injections, which was selected as the earliest time point of preparation and examination of cells. During the further development of pancreatitis almost all acinar cells contain multiple vacuoles (Fig. $1 E-F$ and $K-L$ ). On electronmicrographs these vacuoles contain zymogen granules and other partly digested intracellular compartments $(4,8)$. The vacuoles are an early histological alteration in this model and do not further increase in size and number at time points later than 8-12 $\mathrm{h}$ after start of the cerulein injections. Therefore, the time point of $10 \mathrm{~h}$ after the beginning of the cerulein injections was selected for the latest cell preparation in this model.

Diet-induced pancreatitis. Female Swiss-Webster mice (Simonsen Laboratories), 4-6 wk old and weighing 12-15 g, were used in these studies. The mice had been fed regular laboratory chow before the experiments. This regular laboratory diet was then replaced by a choline-deficient, ethionine-supplemented $(0.5 \%)$ diet (CDE diet; Teklad test diets) that is known to induce a severe hemorrhagic pancreatitis $(1,6-7)$.

Early in the time course of both cerulein- and diet-induced pancreatitis, there was a dramatic decrease in ductal secretion and a concomitant increase in serum of pancreatic enzymes $(1,6-7)$. Also similar to cerulein-induced pancreatitis, appearance of intracellular vacuoles is one of the earliest morphological alterations in this model $(1$, 6-7). After $30 \mathrm{~h}$ of feeding the CDE diet, intracellular vacuoles are the predominant histological feature without major signs of inflammation, necrosis, or hemorrhage, which all appear later in this model (6-8). The time point of $30 \mathrm{~h}$ was, therefore, chosen as the earliest time for preparation and examination of cells. The vacuoles in diet-induced pancreatitis appear similar to those seen in cerulein-induced pancreatitis when assessed by both histological and electron micrograph appearance. As the diet-induced pancreatitis progresses, necrosis and hemorrhage become increasingly predominant. Animals start to die from the hemorrhagic pancreatitis after $60 \mathrm{~h}$ on the diet. This time point was selected as the latest time point at which cells were prepared and examined. At later time points it was not possible to distinguish zymogen granules from intracellular vacuoles due to the huge accumulation of both intracellular compartments.

\section{Preparation of dispersed pancreatic acinar cells}

Dispersed acinar cells were prepared from rat or mouse pancreas at various times after induction of pancreatitis, by previously described techniques $(14,15)$. After sacrifice of the animal, the pancreas was quickly removed and put in Hepes-buffered Ringer solution (HR) (pH 7.4), which was equilibrated with $100 \% \mathrm{O}_{2}$ and kept at $37^{\circ} \mathrm{C}$ throughout the experiments. One pancreas was used for rat experiments; for mouse experiments four to five pancreases were pooled to prepare cells. The same preparative method was then used for rat and mouse pancreas. Dissociation was initiated by injection of $5 \mathrm{ml}$ of HR containing $100 \mathrm{U} / \mathrm{ml}$ collagenase, $10 \mathrm{U} / \mathrm{ml}$ alpha-chymotrypsin, and 100 $\mathrm{U} / \mathrm{ml}$ hyaluronidase into the glandular parenchyma which was then minced into small fragments and incubated in $10 \mathrm{ml} \mathrm{HR}$ containing the dispersion enzymes for $15 \mathrm{~min}$ with shaking at $120 \mathrm{cycles} / \mathrm{min}$. The medium was then replaced with $10 \mathrm{ml}$ of $\mathrm{Ca}^{2+}-\mathrm{Mg}^{2+}$-free HR containing $1.0 \mathrm{mM}$ EDTA, and incubation was continued for $15 \mathrm{~min}$. Pancreatic fragments were then returned to $10 \mathrm{ml} \mathrm{HR}$ containing the dispersion enzymes for a final 20-min incubation. The medium was then replaced by $10 \mathrm{ml}$ of $\mathrm{Ca}^{2+}-\mathrm{Mg}^{2+}$-free $\mathrm{HR}$ containing $2 \mathrm{mg} / \mathrm{ml}$ bovine serum albumin, $0.1 \mathrm{mg} / \mathrm{ml}$ soybean trypsin inhibitor, and $10 \mathrm{U} / \mathrm{ml}$ DNAse. The fragments were dispersed by repeatedly pipetting them with a silicon-treated Pasteur pipette. The cells were filtered through a $50-\mu \mathrm{m}$ nylon mesh, centrifuged for $5 \mathrm{~min}$ at $70 \mathrm{~g}$, and resuspended in $10 \mathrm{ml} \mathrm{HR}$. 90 to $95 \%$ of cells were acinar cells as identified by a cluster of dark refractile granules and 95-100\% excluded trypan blue when prepared from normal pancreas. In the models of acute pancreatitis, in particular in diet-induced hemorrhagic pancreatitis, up to $20 \%$ of cells prepared did not exclude trypan blue, indicating severe damage due to pancreatitis.

\section{Microscopy of acinar cells}

Freshly dispersed acinar cells were incubated until use in oxygenated HR solution (pH 7.4) at $37^{\circ} \mathrm{C}$ for up to $2 \mathrm{~h}$. $\mathrm{AO}(50 \mu \mathrm{M})$ in HR was added to small aliquots of cells (to a final volume of $500 \mu \mathrm{l}$ ), and these were incubated for $2-3 \mathrm{~min}$ at $37^{\circ} \mathrm{C}$. Cells were then placed on a glass slide under a coverslip and visualized. AO is a weak base, which is protonated and concentrated inside acidic compartments where it undergoes a concentration-dependent shift to red-orange fluorescence. Therefore, acidic compartments (e.g., acidic intracellular vesicles) fluoresce red-orange against a green background (e.g., cytoplasm).

Phase contrast light microscopy and fluorescence microscopy were done using a microscope (BH; Olympus Corp. Tokyo, Japan) equipped with a phase-contrast lens and a dichromic B filter, a 0530 barrier, and two BG12 excitation filters for fluorescent microscopy. Phase contrast and fluorescent photomicrographs were taken from the same group of cells with 1 min using an Olympus C 35 camera, a Planapo $40 \times$ or $100 \times$ objective (Zeiss, Oberkochen, FRG), and Ektachrome ASA 400 film (Kodak). In some studies, cells were exposed to various experimental agents (protonophores, $\mathrm{NH}_{4} \mathrm{Cl}$ ), which are known to discharge $\mathrm{pH}$ gradients, before visualization.

\section{Morphometric analysis of percentage area of cytoplasm occupied by zymogen granules and vacuoles}

Fluorescent and phase contrast photomicrograph transparencies taken of the same group of cells were projected onto heavy white bond paper so that the average cell size was $\sim 15 \mathrm{~cm}$ in diameter. The outer cell membrane, as well as the area occupied by zymogen granules or vacuoles (on phase contrast light micrographs), were marked lightly with pencil. The same procedure was then repeated for fluorescent micrographs. The outer cell membrane, as well as the area occupied by zymogen granules and vacuoles (as identified by red-orange compartments in the fluorescent micrographs), were marked lightly with a pencil. For both phase contrast and fluorescent transparencies, the paper area occupied by the whole cells was cut out and weighed; the paper area occupied by the zymogen granules or vacuoles (on phase contrast micrographs) as well as the corresponding red-orange compartments (on fluorescent micrographs) was then cut out of the cell and also weighed. The weight of areas occupied by the zymogen granules or vacuoles and the corresponding red-orange compartments divided by the weight of areas occupied by the whole cells was taken to represent the percentage area of cytoplasm occupied by zymogen granules or vacuoles as identified on phase contrast or on fluorescent micrographs. The morphometric measurements of 50 cells were combined and expressed as mean $\pm \mathrm{SE}$ for each group.

This morphometric analysis yielded highly reproducible results measuring the percentage area occupied by zymogen granules and the corresponding red-orange (acidic) compartments in normal pancreatic cells, with a coefficient of variation of $<1 \%$. In cells from animals with experimental acute pancreatitis, one has not only to identify the zymogen granules and the corresponding red-orange (acidic) compartments, but to also distinguish zymogen granules from vacuoles both in the phase contrast and in the fluorescent micrographs. In cerulein-induced pancreatitis, zymogen granules and vacuoles could easily been identified and distinguished both on phase contrast and fluorescent micrographs with a coefficient of variation of $2-3 \%$ on 20 consecutive measurements. In diet-induced pancreatitis, exact differentiation of zymogen granules from vacuoles could reliably be done only during the first $48 \mathrm{~h}$ of pancreatitis. At this time point, the coefficient of variation increased to 3-5\% due to the massive increase in both zymogen granules and vacuoles that became increasingly difficult to differ- 
entiate as the pancreatitis progressed. After feeding the diet for $60 \mathrm{~h}$, differentiation of zymogen granules and vacuoles was no longer possible. Therefore, for this late time point in diet-induced pancreatitis, only the combined area of zymogen granules plus vacuoles and the combined area of all red-orange compartments were measured on phase contrast and fluorescent micrographs, respectively. This combined measurement, however, yielded highly reproducible results with a coefficient of variation of $1-2 \%$.

In an additional analysis, a regression analysis was carried out comparing the combined areas of zymogen granules and vacuoles identified on phase contrast micrographs versus the combined areas of all red-orange compartments identified on fluorescent micrographs including all time points studied during the course of pancreatitis in both models.

Statistical analysis. Means of morphometric measurements at different times after induction of pancreatitis were statistically compared for significant differences by analysis of variance using Duncan's methods (16-17). Significance was assumed for $P \leq 0.05$.

\section{Results}

Cells from normal mouse and rat pancreas. Fluorescence microscopy of normal rat or mouse acinar cells incubated with 50 $\mu \mathrm{m} \mathrm{AO}$ for 1-2 min showed large red-orange fluorescent compartments in the cytoplasm (rat pancreatic cells are shown in Fig. $1 B$; zymogen granules from mouse pancreatic acinar cells had a similar appearance both on phase contrast and fluorescence micrographs and are not shown). These orange compartments exactly corresponded to areas occupied by zymogen granules identified on the corresponding phase contrast micrographs (Fig. $1 A, B$ ), as previously reported (18). It is known that $\mathrm{AO}$ accumulates inside acidic compartments where it undergoes a concentration-dependent shift to red-orange fluorescence. Therefore, acidic compartments fluoresce red-orange against a green cytoplasmic background. Such orange fluorescence may, however, also be due to nonspecific binding of $\mathrm{AO}$ to cellular structures. In order to establish that orange fluorescence of zymogen granules is due to an acidic interior and not due to nonspecific binding, cells were exposed to agents known to dissipate $\mathrm{pH}$ gradients. Addition of $10 \mu \mathrm{M}$ monensin or 5 $\mu \mathrm{M}$ CCCP completely abolished orange fluorescence in zymogen granules within 1-2 min, as did a 5-10-min exposure to the weak base $\mathrm{NH}_{4} \mathrm{Cl}(10 \mathrm{mM})$ (rat acinar cells in the presence of CCCP are shown in Fig. $1 a, b$; acinar cells isolated from rat or mouse pancreas in the presence of monensin or $\mathrm{NH}_{4} \mathrm{Cl}$ yielded equivalent images and are not shown) (18). Previous studies (18) have shown that this acidification of zymogen granules is due to a reversible, active, and ATP-dependent process similar to that reported for proton pumps in other secretory or endocytic vesicles (19-21).

Cerulein-induced pancreatitis. Throughout the time course of cerulein-induced pancreatitis, intracellular vacuoles could easily be identified on the phase contrast micrograph; and they exactly corresponded to orange acidic (vacuole-like) compartments shown on the corresponding fluorescent micrographs (Fig. $1 C-F$ ). Also, the vacuoles could reliably be distinguished from zymogen granules both on phase contrast and on fluorescent micrographs in this pancreatitis model. Intracellular vacuoles increased in size and number during the first $10 \mathrm{~h}$ of cerulein-induced pancreatitis. $3 \mathrm{~h}$ after the start of the cerulein injections vacuoles occupied $\sim 5 \%$ of the cytoplasmic area (Fig. $2 \mathrm{~A}$ ). This area increased to $10 \%$ after $6 \mathrm{~h}$ and $15 \%$ after $10 \mathrm{~h}$ (Fig. $2 \mathrm{~A}$ ). Later in the time course there was no further increase (data not shown). The morphometric analysis also demonstrates that the cytoplasmic area covered by vacuoles on phase contrast micrographs exactly corresponded to the areas of vacuoles identified as red-orange acidic compartments on fluorescent micrographs throughout the time course of pancreatitis (Figs. $2 A$ and 3 ). This indicates that all vacuoles remained acidic during the early course of cerulein-induced pancreatitis.

Zymogen granules were acidic in cells isolated from both normal rats and from cerulein-treated animals. In contrast to the vacuoles, there was no significant increase in the cytoplasmic area covered by zymogen granules during the course of cerulein-induced pancreatitis (Fig. $1 C-F$ ). The area occupied by zymogen granules as identified on phase contrast micrographs exactly corresponded to the area occupied by red-orange granules identified on fluorescent micrographs and remained constant throughout the time course of cerulein-induced pancreatitis covering between 13 and $15 \%$ of cytoplasm (Fig. $2 \mathrm{~A}$ ). Thus, all zymogen granules remained acidic as the pancreatitis progressed.

Diet-induced pancreatitis. In diet-induced hemorrhagic pancreatitis, the vacuoles seen on phase contrast micrograph again exactly corresponded to vacuole-like, orange acidic compartments on the fluorescent micrographs throughout the early time course (Fig. $1 G-H$ ). Vacuoles were absent in normal cells and gradually increased to $15 \%$ of cytoplasmic area after $48 \mathrm{~h}$ (Fig. $2 \mathrm{~B}$ ). Up to this time point the percentage area of cytoplasm covered by vacuoles was identical when identified on phase contrast micrographs and when identified as red-orange vacuoles on fluorescence micrographs (Figs. $2 B$ and 3).

In diet-induced pancreatitis, there was a significant intracellular accumulation of zymogen granules (Figs. $1 \mathrm{G}-\mathrm{H}, 1$ $K-L, 2 B$, and 3 ), as previously reported $(1,6-8)$. The percentage area of zymogen granules on phase contrast micrographs, however, remained identical compared to the area covered by the corresponding red-orange granules (Figs. $2 B$ and 3). This indicates that all accumulating zymogen granules remained acidic throughout the time course of diet-induced pancreatitis.

Later during the time course of diet-induced pancreatitis (time points later than $48 \mathrm{~h}$ after start of the diet), there was a further accumulation of both zymogen granules and vacuoles inside the acinar cells (Fig. $1 K-L$ ). Due to this further increase in both compartments, it became increasingly difficult to distinguish zymogen granules from vacuoles. $60 \mathrm{~h}$ after start of the diet, it was impossible to reliably distinguish zymogen granules from vacuoles both on phase contrast and on fluorescent micrographs (Fig. $1 K-L$ ). Both compartments taken together occupied $>50 \%$ of cytoplasm (Fig. $2 \mathrm{~B}$ ). The combined area occupied by zymogen granules and vacuoles was identical when measured on phase contrast micrographs and when measured on fluorescent micrographs (Fig. $2 B$ and 3), indicating that all zymogen granules and vacuoles remained acidic late in the development of diet-induced pancreatitis.

Regression analyses. Previously, morphometric analysis of acinar cells from fasted rats and from rats after in vivo cholinergic stimulation had shown a tight linear regression of compartments occupied by zymogen granules versus AO-positive compartments (18). The present regression analysis comparing . the combined areas occupied by zymogen granules and vacuoles on phase contrast micrographs versus the orange-acidic areas on fluorescent micrographs demonstrates a tight linear 


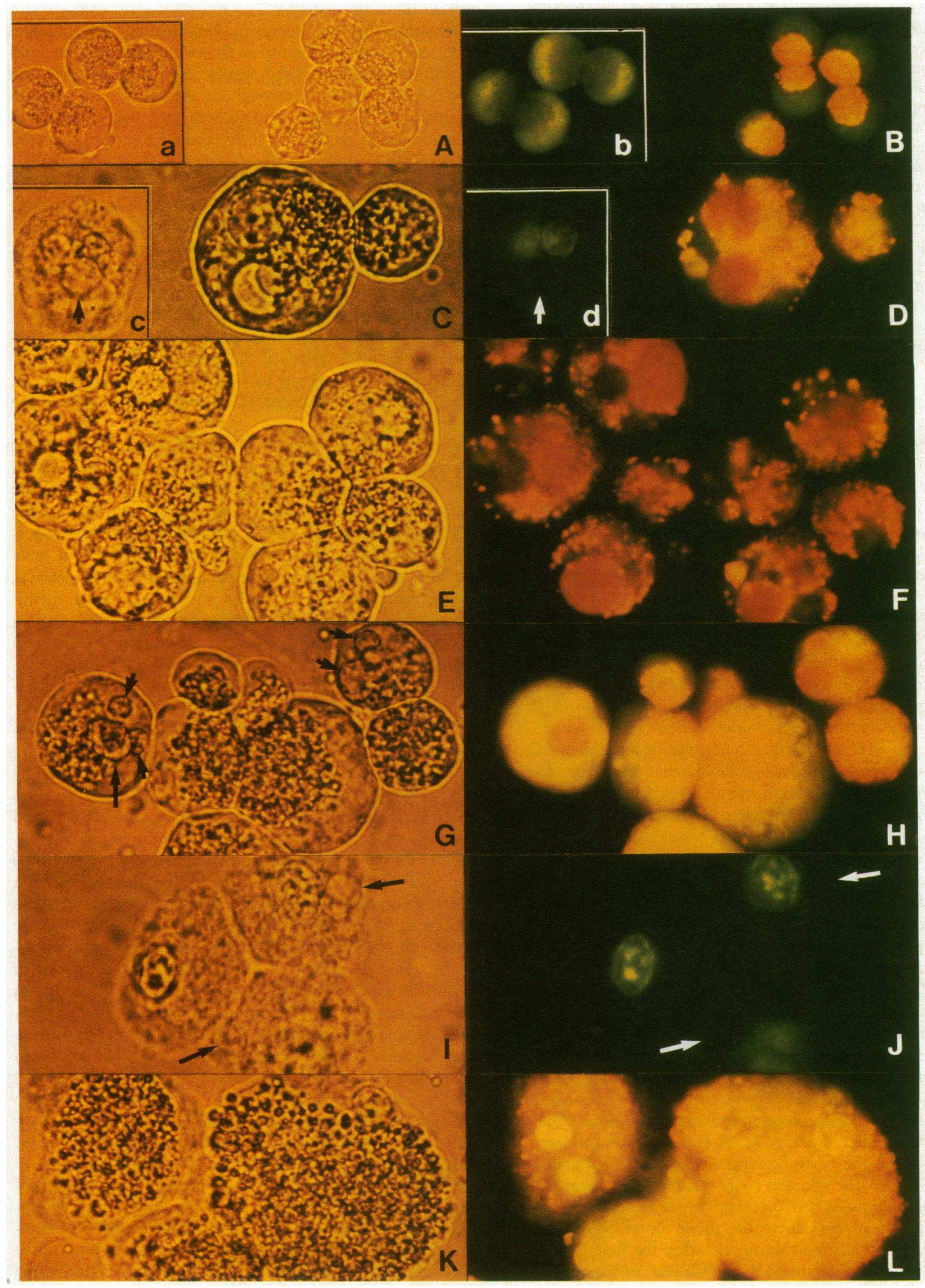


correlation, indicating that both zymogen granules and vacuoles remained acidic throughout the time course in both models; the same very tight linear regression was found when previous morphometric data from fasted rats and from rats after in vivo cholinergic stimulation (18) were included in the present analysis ( $r=0.98$ ) (Fig. 3). Regression analyses also showed a tight linear correlation when calculated separately for zymogen granules or vacuoles or when calculated separately for diet-induced or cerulein-induced pancreatitis ( $r$ $>0.90$ ) (data not shown).

Control studies using protonophores and $\mathrm{NH}_{4} \mathrm{Cl}$. Because it is known that acridine orange may bind to cell structures by mechanisms other than pH gradients, it is necessary to exclude nonspecific binding by addition of compounds that dissipate $\mathrm{pH}$ gradients. We used the protonophores monensin and CCCP that rapidly discharge $\mathrm{pH}$ gradients (monensin by exchange of $\mathrm{K}^{+}$or $\mathrm{Na}^{+}$against $\mathrm{H}^{+}$and $\mathrm{CCCP}$ by electrogenic movement of $\mathrm{H}^{+}$across the membrane of vesicles), and the weak base $\mathrm{NH}_{4} \mathrm{Cl}$ that dissipate $\mathrm{pH}$ gradients by diffusion of $\mathrm{NH}_{3}$ into acidic compartments and protonation to $\mathrm{NH}_{4}^{+}$.

As previously demonstrated (18), AO fluorescence in zymogen granules from normal pancreatic cells was completely dissipated by protonophores and $\mathrm{NH}_{4} \mathrm{Cl}$ (Fig. $1 a-b$ ). In both pancreatitis models, these compounds also rapidly and completely dissipated acridine orange fluorescence both in zymogen granules and in vacuoles throughout the time course of pancreatitis (Fig. $1 c$ - $d$ and $I-J$ ).

\section{Discussion}

The present study provides direct evidence that intracellular vacuoles in two different models of acute pancreatitis are an acidified compartment. Throughout the time course of both pancreatitis models, the intracellular area occupied by vacuoles as identified on phase contrast micrographs corresponded exactly to that area that was covered by the red-orange vacuoles as identified on fluorescent micrographs. This was already apparent by visualizing the localization of vacuoles by phase contrast microscopy and visualizing the localization of the corresponding red-orange, vacuole-like compartments by fluorescence microscopy and was further corroborated by the quantitative morphometric analysis done on phase contrast and fluorescent micrographs of the same cells.

The present results also show that zymogen granules remain acidic throughout the time course of pancreatitis in both models. The morphometric analysis quantifies and differentiates the accumulation of zymogen granules and vacuoles in both models of pancreatitis, a feature not previously described. Although the accumulation of vacuoles was very similar for the two different models, a significant accumulation of zymogen granules was seen only in diet-induced pancreatitis.

Control experiments showed that compounds that discharge $\mathrm{pH}$ gradients (monensin by exchange of $\mathrm{K}^{+}$or $\mathrm{Na}^{+}$for $\mathrm{H}^{+}$; CCCP by electrogenic movement of $\mathrm{H}^{+}$across the membrane; and $\mathrm{NH}_{4} \mathrm{Cl}$, which diffuses into the acidic compartment and acts as a proton acceptor) rapidly and completely abolished AO fluorescence both in zymogen granules and vacuoles throughout the time course in both models of acute pancreatitis. This indicates that accumulation of $\mathrm{AO}$ in both zymogen granules, as shown previously (18), and in vacuoles is indeed due to an acidic interior and not due to nonspecific binding of AO to some structural component of these intracellular compartments.

The appearance of vacuoles inside acinar cells character-
Figure 1. Paired phase contrast and fluorescent photomicrographs taken from the same group of cells within $1 \mathrm{~min}$. An Olympus $C 35$ mm camera, a Planapo $40 \times$ objective $(a-b$ and $A-B)$ or $100 \times$ objective $(C-L)$, and Ektachrome ASA 400 film were used. ( $A$ and $B$ ) Freshly dispersed acinar cells from normal rat pancreas. Note that the cluster of dark refractile granules on the phase contrast micrograph $(A)$ exactly corresponds to the red-orange areas on the fluorescent micrograph $(B)$. Shown as inserts $(a)$ and $(b)$ are cells that were incubated for 1-2 min with $50 \mu \mathrm{M}$ AO in the presence of $5 \mu \mathrm{M}$ CCCP. Note that the presence CCCP completely abolished AO fluorescence $(b)$ in the area occupied by zymogen granules $(a)$. Monen$\sin \left(10 \mu \mathrm{M}\right.$ and $\left.\mathrm{NH}_{4} \mathrm{Cl}(10 \mathrm{mM})\right)$ similarly dissipated $\mathrm{AO}$ fluorescence (images were equivalent to those in the presence of $5 \mu \mathrm{M}$ CCCP and are not shown). Acinar cells isolated from normal mouse pancreas yielded equivalent images compared to acinar cells isolated from normal rat pancreas and are not shown. ( $C$ and $D$ ) Rat pancreatic acinar cells isolated $6 \mathrm{~h}$ after the start of the cerulein injections. Large vacuoles can easily be identified on the phase contrast micrograph $(C)$ that correspond to orange acidic vacuoles on the corresponding fluorescent micrograph of the same group of cells $(D)$. $\mathrm{Zy}-$ mogen granules also show AO fluorescence $(D)$ indicating that they remained acidic. Shown as inserts $(c)$ and $(d)$ are cells that were incubated for 1-2 min with $50 \mu \mathrm{M}$ AO in the presence of $5 \mu \mathrm{M} \mathrm{CCCP.}$ Note that the presence of $\mathrm{CCP}$ completely abolished AO fluorescence in vacuoles as seen on the phase contrast micrograph $(b)$ and on the corresponding fluoresencent micrograph (c) (arrows indicate large vacuoles). Monensin $(10 \mu \mathrm{M})$ and $\mathrm{NH}_{4} \mathrm{Cl}(10 \mathrm{mM})$ also completely dissipated $\mathrm{AO}$ fluorescence in zymogen granules and in vacuoles (images were equivalent to those shown for $5 \mu \mathrm{M} \mathrm{CCCP}$ and are not shown). ( $E$ and $F$ ) Rat pancreatic acinar cells isolated $10 \mathrm{~h}$ after the start of the cerulein injections. Almost all cells contain large and often multiple vacuoles as identified on the phase contrast micrograph $(E)$ all of which show AO fluroescence on the corresponding fluorescent micrograph $(F)$. Zymogen granules continue to show AO fluorescence at this time point in cerulein-induced pancreatitis. There is no apparent increase in the cytoplasmic area occupied by zymogen granules that appear to be scattered inside the cell rather than oriented towards one portion of the cell as seen in normal cells (see $A$ and $B$ for comparison). ( $G$ and $H$ ) Mouse pancreatic acinar cells isolated $30 \mathrm{~h}$ after the start of the CDE diet. At this early time point in the development of diet-induced pancreatitis, large cytoplasmic vacuoles are seen on the phase contrast micrograph (arrows) $(G)$ and these vacuoles show $\mathrm{AO}$ fluorescence on the corresponding fluorescent micrograph $(H)$. Already at this early time point there is also an apparent increase in the cytoplasmic area occupied by zymogen granules (see $A$ and $B$ for comparison) ( $I$ and $J$ ) Mouse pancreatic acinar cells isolated $30 \mathrm{~h}$ after the start of the CDE diet. Cells were incubated for 1-2 min in $\mathrm{HR}$ with $50 \mu \mathrm{M}$ AO in the presence of 5 $\mu \mathrm{M}$ CCCP. AO fluorescence is completely abolished by CCCP both in zymogen granules as well as in vacuoles (arrows indicate large vacuoles). Monensin $(10 \mu \mathrm{M})$ and $\mathrm{NH}_{4} \mathrm{Cl}(10 \mathrm{mM})$ also completely dissipated $\mathrm{AO}$ fluorescence in zymogen granules and vacuoles inside cells isolated $30 \mathrm{~h}$ after the start of the CDE diet (images in the presence of monensin or $\mathrm{NH}_{4} \mathrm{Cl}$ were equivalent to images obtained with CCCP and are not shown). ( $K$ and $L$ ) Mouse pancreatic acinar cells isolated $60 \mathrm{~h}$ after the start of the CDE diet. Due to the massive increase in both zymogen granules and vacuoles, which occupy the majority of cytoplasmic area at this late time point in the course of pancreatitis, it is impossible to exactly distinguish both compartments either on the phase contrast micrograph $(K)$ or on the corresponding fluorescent micrograph $(L)$. However, it is apparent that both zymogen granules and vacuoles show AO fluorescence $(L)$. 

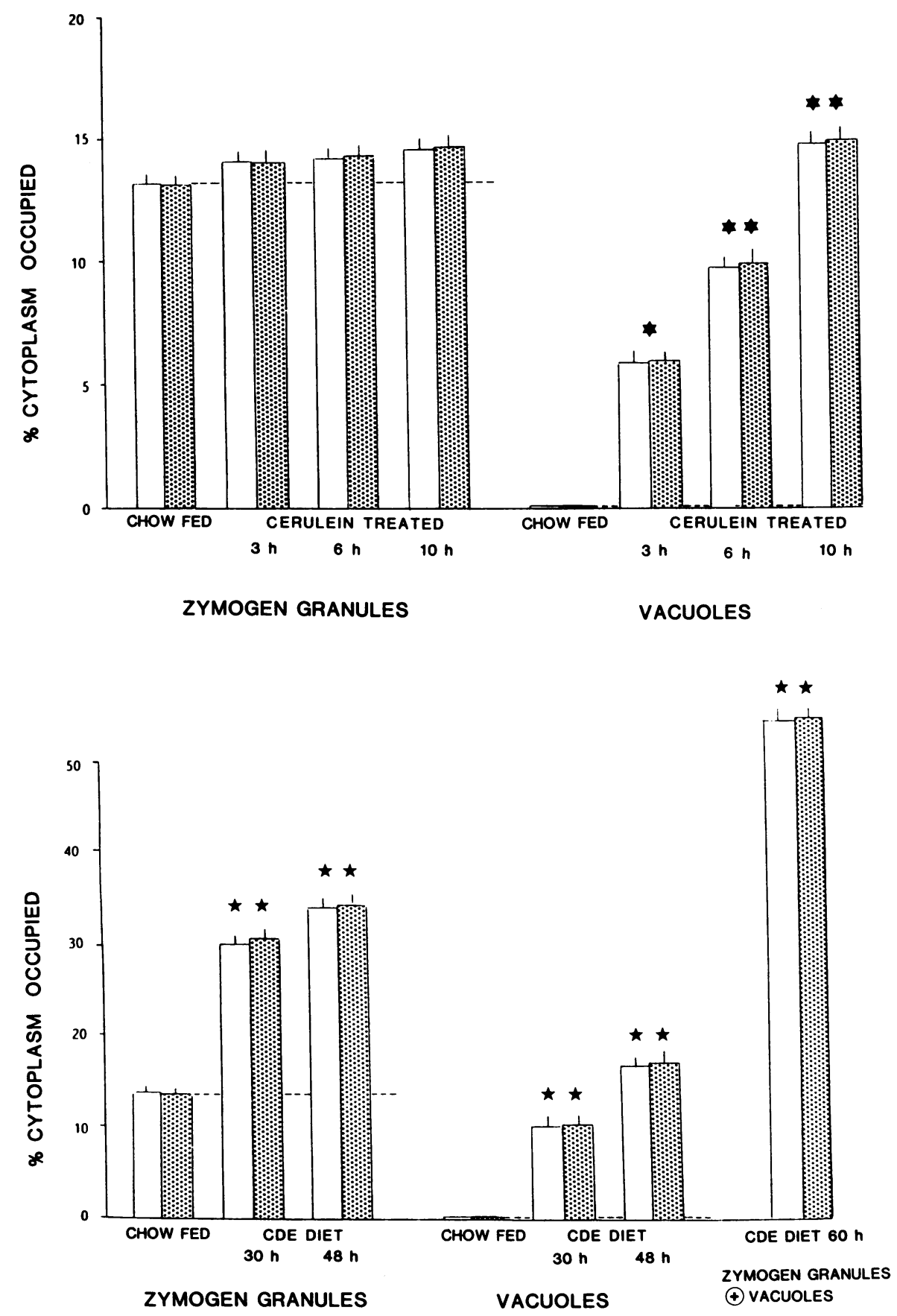

Figure 2. (A and B) Percentage area of cytoplasm occupied by zymogen granules or vacuoles was measured as described in the Method section. Open bars indicate measurements on phase contrast micrographs and shaded bars indicate measurements on fluorescence micrographs. All measurements were done in 50 cells for each condition and time point and are given as mean \pm SE. In all instances, the areas identified on phase contrast micrographs were identical with the corresponding red-orange, acidic compartments identified on fluorescent micrographs $(P>0.2$ by analysis of variance). ( $A$ ) Cerulein-induced pancreatitis. There was no significant increase in the cytoplasmic area occupied by zymogen granules during the time course of cerulein-induced pancreatitis compared to chow-fed control values $(P>0.2$ by analysis of variance). However, there was a significant, time-dependent increase in the cytoplasmic area occupied by vacuoles (*indicates $P \leq 0.01$ and ${ }^{* *}$ indicates $P \leq$ 0.001 when results were compared to results in chow-fed animals by analysis of variance). The area occupied by vacuoles at $6 \mathrm{~h}$ was significantly higher compared to the value at $3 \mathrm{~h}(P \leq 0.05$ by analysis of variance) and the value at $10 \mathrm{~h}$ was significantly higher compared to the value at $3 \mathrm{~h}$ $(P \leq 0.001)$ and compared to the value at $6 \mathrm{~h}(P \leq 0.05)$. (B) Diet-induced pancreatitis. There was a significant increase in the percentage area occupied by zymogen granules during the time course of pancreatitis $\left({ }^{* *} P \leq 0.001\right.$ when the values at $30 \mathrm{~h}$ or $48 \mathrm{~h}$ were compared to the control values in chow fed animals by analysis of variance). There was also a significant, time-dependent increase in the area occupied by vacuoles during the development of diet-induced pancreatitis ${ }^{* *} P \leq 0.001$ when results were compared to control values in chow-fed animals by analysis of variance). The area occupied by vacuoles at $48 \mathrm{~h}$ was significantly higher compared to the area at $30 \mathrm{~h}(P \leq 0.01$ by analysis of variance). At later time points, such as 60 $h$ it became impossible to distinguish zy-

mogen granules from vacuoles. Therefore, only the combined area of both compartments could be measured (bars on the far right) $\left({ }^{* *} P \leq\right.$ 0.001 when the results of combined measurements of zymogen granules and vacuoles at $60 \mathrm{~h}$ were compared with control value of combined measurements in chow-fed animals by analysis of variance).

izes an early stage of development in different animal models of acute pancreatitis (1-8) and possibly also in human disease (9). Electron micrographs show that these vacuoles contain zymogen granules and other partly digested cell compartments $(3-6,8)$. It has also been shown that the vacuoles may at the same time contain lysosomal enzymes $(5,10-12)$. This indicates that intracellular processing of newly synthesized digestive and lysosomal enzymes may be altered in acute pancreatitis. Physiologically, digestive and lysosomal enzymes are synthesized in ribosomes attached to the rough endoplasmic reticulum. They migrate through this compartment and are transported to the Golgi complex. During maturation of condensing vacuoles, the digestive and lysosomal enzyme diverge into zymogen granules and lysosomes. In the two models of pancreatitis used for the present experiments this normal routing of enzymes is indeed altered. Both in diet-induced hemorrhagic pancreatitis in mice, and in edematous pancreatitis induced by cerulein hyperstimulation in rats, normal secretion of digestive enzymes via exocytosis of zymogen granules is markedly decreased and intracellular vacuoles ap- 


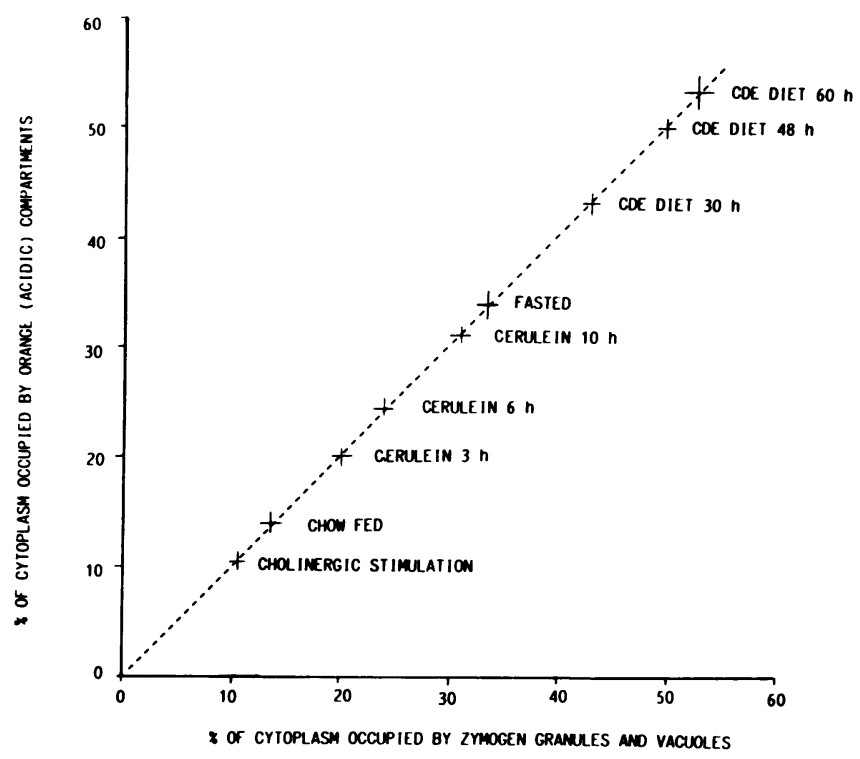

Figure 3. Regression analysis comparing the combined areas occupied by zymogen granules plus vacuoles in both cerulein-induced pancreatitis and in diet-induced pancreatitis. Measurements and calculations were carried out at various time points during the course of pancreatitis as indicated in the figure and described in Methods. Data previously obtained by the same morphometric analysis of compartments occupied by zymogen granules versus AO-positive compartments in acinar cells from fasted rats and from rats after in vivo cholinergic stimulation (18) were included in the present analysis. Values are shown as mean $\pm \mathrm{SE}$ of measurements of 50 cells for each time point and condition. The regression analysis revealed a tight linear relation with $r=0.98(P \leq 0.001)$ and a slope that was 0.99 .

pear early in the course of pancreatitis (1-8, 10-12). Vacuole formation in diet-induced pancreatitis has been explained by an abnormal fusion of zymogen granules with lysosomes called crinophagy $(6,12)$, whereas in cerulein-induced pancreatitis there may be an abnormal maturation of the condensing vacuole $(5,12)$ as well as fusion of several zymogen granules (22) and possibly also fusion of vacuoles and zymogen granules with the basolateral membrane (22). Vacuoles in both models may contain both digestive enzymes and lysosomal hydrolases $(5,10-12)$. This abnormal admixture of digestive and lysosomal enzymes may have important implications for the initiation of pancreatitis because it is known that the lysosomal hydrolase cathepsin B can activate trypsinogen in vitro (13). In addition it has been shown that endogenous cathepsin B can activate trypsinogen in extracts of dog pancreas (23). Furthermore, inhibition of cathepsin B in pancreatic tissue was associated with marked amelioration of pancreatitis in the diet model (24). Thus, activation of trypsinogen in vivo may trigger the autodigestive process in acute pancreatitis. Cathepsin B can activate trypsinogen, however, only at an acidic pH (13). The present results give direct evidence that the $\mathrm{pH}$ inside these vacuoles is indeed acidic. Because AO fluorescence does not increase linearly in acidic compartments in response to decreasing $\mathrm{pH}$ values it is not possible to use $\mathrm{AO}$ to determine the precise $\mathrm{pH}$ in an organelle. However, the vacuoles observed in these models of acute pancreatitis fluoresce similarly to hepatic lysosomes and other intracellular organelles containing a proton pump. The internal $\mathrm{pH}$ of these or- ganelles has been quantified by other means and approaches the $\mathrm{pH}$ range of 4 to 5 necessary for activation of trypsinogen by cathepsin B (19-21).

It appears remarkable that the intracellular vacuoles behave very similarly in both models of pancreatitis that are quite different in several respects. First, two different species were used for the two models. Second, the CDE diet produces a severe hemorrhagic pancreatitis. The morphological lesions seen in this model are similar to what is seem in severe human hemorrhagic-necrotizing pancreatitis; and this model is also associated with a high mortality $(1,7)$. As in human hemorrhagic pancreatitis, metabolic acidosis, hypoxemia, and dehydration are complications in the diet-induced pancreatitis (25). In contrast, hyperstimulation by supramaximal doses of cerulein leads to edematous pancreatitis. The morphological lesions are similar to what is seen in human edematous pancreatitis, and similarly to human edematous pancreatitis this model is not associated with any significant mortality (2-5). Third, the pathogenesis of the two models used appears to be completely different.

A number of recent observations lend further support to the hypothesis that intracellular vacuolization is a general feature in the development of acute pancreatitis and, therefore, may have important pathophysiological implications for the human disease. Recently, we have studied the early morphological changes in acute hemorrhagic-necrotizing pancreatitis in sheep by retrograde infusion of the bile salt sodium taurocholate into the pancreatic duct (8), similarly to the rat model described previously (26). In this model large areas of the pancreas rapidly undergo hemorrhagic necrosis. In the remaining viable acinar cells surrounding the totally necrotic areas contain multiple large intracellular vacuoles that appear very similar to the vacuoles observed in the course of diet-induced or cerulein-induced pancreatitis in the rat or mouse. Thus, in acute pancreatitis produced in three different species by three different means, a similar vacuolization process occurs (1-12). In addition, recent studies by Klöppel et al. (9) have demonstrated intracellular vacuoles during the early course of acute pancreatitis in humans. Thus, an understanding of the characteristics of these vacuoles may yield important information concerning the pathophysiology of clinical acute pancreatitis.

As yet it has not been shown that the intracellular vacuoles from patients with acute pancreatitis also contain both digestive and lysosomal enzymes at an acidic pH. However, the striking similarities in appearance suggest that this may be the case. What also remains to be demonstrated is direct evidence that trypsinogen is activated by cathepsin $B$ in vivo inside vacuoles in acute pancreatitis. However, the present results support the hypothesis that such activation occurs and suggest the need for further studies to investigate the potential for intrapancreatic activation of digestive enzymes by lysosomal enzymes within vacuoles as a central event in the development of acute pancreatitis.

\section{Acknowledgments}

Dr. Niederau was supported by grants from the Deutsche Forschungsgemeinschaft (Ni 224/1-1 and 2-1).

\section{References}

1. Lombardi, B., L. W. Estes, and D. S. Longnecker. 1975. Acute hemorrhagic pancreatitis (massive necrosis) with fat necrosis induced 
in mice by DL-ethionine with a choline-deficient diet. Am. J. Pathol. 79:464-480.

2. Lampel, M., and H. F. Kern. 1977. Acute interstitial pancreatitis induced in the rat by excessive doses of a pancreatic secretagogue. Virchows Arch. (A). 373:97-117.

3. Adler, G., T. Hupp, and H. F. Kern. 1979. Course and spontaneous regression of acute pancreatitis in the rat. Virchows Arch. (A). 882:32-47.

4. Niederau, C., L. D. Ferrell, and J. H. Grendell. 1985. Caeruleininduced acute necrotizing pancreatitis in mice: protective effects of proglumide, benzotript, and secretin. Gastroenterology. 88:11921204.

5. Watanabe, O., F. M. Baccino, M. L. Steer, and J. Meldolesi. 1984. Supramaximal caerulein stimulation and ultrastructure of rat pancreatic acinar cell: early morphological alterations during development of experimental pancreatitis. Am. J. Physiol. 246:G457-G467.

6. Koike, K., M. L. Steer, and J. Meldolesi. 1982. Pancreatic effects of ethionine: blockade of exocytosis and appearance of crinophagy and autophagy preceding cellular necrosis. Am. J. Physiol. 242:G297G307.

7. Niederau, C., R. A. Liddle, L. D. Ferrell, and J. H. Grendell. 1986. Beneficial effects of cholecystokinin-receptor blockade and inhibition of proteolytic enzyme activity in experimental acute hemorrhagic pancreatitis in mice. J. Clin. Invest. 78:1056-1063.

8. Niederau, C., L. D. Ferrell, and J. H. Grendell. 1986. Experimentelle akute Pankreatitis. Internist. 27:681-696.

9. Klöppel, G., T. Dreyer, S. Willemer, H. F. Kern, and G. Adler. 1986. Human acute pancreatitis: its pathogenesis in the light of immunocytochemical and ultrastructural findings in acinar cells. Virchows Arch. (A). 409:791-803.

10. Adler, G., C. Hahn, H. F. Kern, and K. N. Rao. 1985. Cerulein-induced pancreatitis in rats: increased lysosomal enzyme activity and autophagocytosis. Digestion. 32:10-18.

11. Rao, K., N. Zuretti, F. M. Baccino, and B. Lombardi. 1980. Acute hemorrhagic pancreatitis in mice. The activity of lysosomal enzymes in the pancreas and liver. Am. J. Pathol. 98:45-60.

12. Steer, M., J. Meldolesi, and C. Figaralla. 1984. Pancreatitis. The role of lysosomes. Dig. Dis. Sci. 29:934-938.

13. Greenbaum, L. M., A. Hirshkowitz, and I. Shoichet. 1959. The activation of trypsinogen by cathepsin B. J. Biol. Chem. 234:28852890.

14. Williams, J. A., C. P. Cary, and B. Moffat. 1976. Effects of ions on amylase release by dissociated pancreatic acinar cells. Am. J. Physiol. 231:1562-1567.

15. Hootman, S. R., S. A. Ernst, and J. A. Williams. 1983. Secretagogue regulation of $\mathrm{Na}^{+}-\mathrm{K}^{+}$pump activity in pancreatic acinar cells. Am. J. Physiol. 245:G339-G346.

16. Duncan, D. B. 1957. Multiple range-tests for correlated and heteroscedastic means. Biometrics. 13:164-176.

17. Byrkit, D. R. 1980. Analysis of variance. In Elements of Statistics. D. R. Byrkit, editor. D. Van Nostrand, New York. 305-358.

18. Niederau, C., R. W. Van Dyke, B. F. Scharschmidt, and J. H. Grendell. 1986. Rat pancreatic zymogen granules. An actively acidified compartment. Gastroenterology. 91:1433-1442.

19. Ohkuma, S., Y. Moriyama, and T. Takano. 1982. Identification and characterization of a proton pump on lysosomes by fluorescein isothiocyanate-dextran fluorescence. Proc. Natl. Acad. Sci. USA. 79:2758-2762.

20. Cidon, S., H. Ben-David, and N. Nelson. 1983. ATP-driven proton fluxes across membranes of secretory organelles. J. Biol. Chem. 258:11684-11688.

21. Yamashiro, D. J., S. R. Fluss, and F. R. Maxfield. 1983. Acidification of endocytic vesicles by an ATP-driven proton pump. J. Cell Biol. 97:929-934.

22. Adler, G., G. Rohr, and H. F. Kern. 1982. Alteration of membrane fusion as a cause of acute pancreatitis in the rat. Dig. Dis. Sci. 27:993-1002.

23. Greenbaum, L. A., and A. Hirshkowitz. 1961. Endogenous cathepsin activates trypsinogen in extracts of dog pancreas. Proc. Soc. Exp. Biol. Med. 107:74-76.

24. Lombardi, B., and K. N. Rao. 1982. Acute hemorrhagic pancreatic necrosis in mice. Effects of proteinase inhibitors on its induction. Digestion. 23:57-64.

25. Crass, R. C., C. Niederau, L. D. Ferrell, and J. H. Grendell. 1985. The effects of hydration and oxygenation on experimental acute pancreatitis in mice. Gastroenterology. 88:A1518.

26. Lankisch, P. G., K. Winckler, M. Bokermann, H. Schmidt, and W. Creutzfeldt. 1974. The influence of glucagon on acute experimental pancreatitis in the rat. Scand. J. Gastroenterol. 9:725-729. 